\title{
Development and Application of Artificial Intelligence Multimedia Technology Based on Big Data
}

\author{
Yezi Zhang $(1)$ \\ The Catholic University of Korea Graduate School, Seoul 14662, Republic of Korea \\ Correspondence should be addressed to Yezi Zhang; zhangyezi2020@gmail.com
}

Received 20 August 2021; Revised 8 October 2021; Accepted 25 October 2021; Published 4 January 2022

Academic Editor: Sang-Bing Tsai

Copyright (c) 2022 Yezi Zhang. This is an open access article distributed under the Creative Commons Attribution License, which permits unrestricted use, distribution, and reproduction in any medium, provided the original work is properly cited.

\begin{abstract}
Artificial intelligence is an innovative enterprise theme that combines computer science, physiology, science, and other disciplines, including expert programs, technical experiments, games, native language understanding, cognitive rehabilitation, robotics, engineering, recognition, and other fields. The breakthrough growth made the arrival of the era of big data have a fundamental impact on many traditional industries and has also promoted the transformation of teaching methods and methods in the field of education. In the traditional classroom, due to the limitations of teaching resources, teaching time, and location, students are basically passively accepted by the teacher, and the teaching form is single. This will improve in the age of big data with well-developed network message. Modern distance education uses advanced technology such as computer networks, multimedia, and artificial intelligence to build a virtual teaching environment based on the network. It breaks through the limitations of teachers, teaching materials, experimental equipment, and other resources that exist under the traditional teaching model. Using educational resources of the same scale can expand educational capabilities exponentially and at the same time overcomes the unified progress of traditional teaching methods, single teaching methods, and students' dissatisfaction. The shortcomings of differences and personalities that are not reflected are conducive to the improvement of the quality of education. It is the original intention of education to offer students special study resources and to stimulate students' enthusiasm for learning. To enhance the current learning environment, the article builds a personalized multimedia network teaching system based on big data and streaming media technology. By recording the teacher's class process as a video and uploading it to the multimedia network teaching system, students can watch and download related videos online for learning and also communicate online. The system can also provide personalized and intelligent recommendations for students based on student learning habits. The system is developed based on intelligent multimedia method and has the characteristics of security and stability and has done some help for network teaching of educational institutions.
\end{abstract}

\section{Introduction}

This model covers a wide range of multimedia, from simple emails and mailing lists to large and complex learning systems. This mode is generally set up by an educational institution (such as a middle school, university, or research institution) to set up some problems suitable for specific students to solve and publish to students through intelligent multimedia and ask students to answer them. At the same time, a large number of problem-related information resources are provided for students to consult in the process of solving problems. With the rapid development of information technology in China, the importance of information has become increasingly prominent. As an important part of information technology, multimedia technology has been applied to classroom teaching, but this still canno't meet the modern personalized teaching needs, and online learning can make up for this shortcoming to some extent $[1,2]$. In the process of education, we hope that students can conduct a variety of independent learning. For example, various types of teaching software on the Internet are important ways for students to learn independently. However, in the era of explosive growth of information data, it is extremely difficult to obtain the parts that are needed from the vast amount of data [3]. In order to make the information of the Internet more extensive, shared, and targeted, intelligent multimedia 
technology based on big data should be added to it. This will not only provide strong technical support for teacher teaching and student studying but also give complete play to the rationality of learning resources as well $[4,5]$. Therefore, the intelligent multimedia technology based on big data to construct a personalized multimedia online teaching system is in line with the latest requirements of the big data era and is of great significance. In modern distance education, not only can students ask questions to the best teachers, but also the system can automatically give answers to students' questions. In the automatic question feedback and answering system, a database system with an intelligent search engine is used. The representativeness and universality encountered by the students in the learning process, as well as the answers to the questions carefully selected by the teacher, are independently stored in the database in the form of data. When a student asks a question, the system starts an intelligent search engine to automatically find the most comprehensive answer in the database. This function of modern distance education saves the time of students and teachers and improves the utilization of learning resources.

Real-time distance teaching is a one-to-one or one-tomany distance teaching method on the public multimedia communication network. In this teaching method, information such as the voice, images, blackboard writing, and data of the teacher's lecture can be transmitted from the main studio to the remote multimedia classroom where the students are located in real time. At the same time, students' feedback information can also be sent back to teachers from all over the place, and students can also communicate with each other. The whole process is as if students are listening to the teacher in the classroom, so it is also called online virtual classroom. At present, teaching in combination with multimedia is a common phenomenon in many schools. Although the existing multimedia technology is auxiliary in actual teaching, it never necessarily means that the method can meet all teaching needs well. Many related institutions and experts at home and abroad have been committed to the innovation of multimedia teaching. Nowadays, both developed and developing countries attach great importance to the development and utilization of multimedia technology, which can be seen from the rising proportion of investment in multimedia technology in teaching [6]. Europe and USA have begun multimedia teaching very early, and digital virtual technology has now been introduced into teaching [7]. Online classes and webinars are also the first to start from abroad, and now the penetration rate is very high, covering all education systems. Some countries represented by Japan pay special attention to the development and application of multimedia live broadcast technology. They apply this technology not only to school education, but also to some fields, such as administration [8], enterprise [9], and hospitals [10]. China's multimedia started late in teaching, but this does not affect the enthusiasm of domestic experts and scholars for the development and application of technology. In terms of the development status of multimedia technology in higher vocational colleges, Chen and Liu [11] used computer multimedia technology to assist study and effectively improved the level of modern study. Wang [12] integrated multimedia information technology into the teaching process of junior high school English learning. Through reasonable use at the right time, the interest and effectiveness of junior high school English teaching and learning have been greatly improved. Binbin [13] uses multimedia technology to teach in the English class and combines the characteristics of the subject to optimize the teaching, create a situation with multimedia, give students the freshest learning experience of each sense, and then achieve the enhancement of teaching effect. In the context of the data age, Chen [14] followed the pace of educational reform and introduced multimedia technology into high school physics teaching, which improved the dilemma of physics classroom knowledge that was not vivid enough and was recognized and praised by schools, teachers, and students. The teaching methods of various schools are constantly changing, and the same is true in the teaching of chemistry. Wang [15] relied on the visual advantages of multimedia in graphic images, applied multimedia on high school chemistry lessons [16, 17], and evolved abstract teaching into image teaching, which was used as an opportunity to improve the better enhancement of high school chemistry. Yue [18] combined multimedia technology with the naughty students and used multimedia technology in primary school music teaching. Through the use of strengths and weaknesses, the scientific and rational use of multimedia technology has effectively improved the quality of music classroom teaching.

Teachers can understand the students' learning progress and the degree of understanding of the curriculum in time and answer the questions raised by the students. Modern distance education has given new connotations to the concept of teaching and learning, brought profound changes to education, and promoted the renewal of educational concepts, educational thoughts, educational models, and teaching methods. Of course, the modern distance education method is not an omnipotent education method, it cannot completely replace face-to-face instruction, and it is not necessarily applicable to all educational levels and courses. It requires students to have strong self-learning ability and selfconsciousness. The use of computer Internet for distance education has now attracted the attention of many countries and people, and our country is stepping up its construction and implementation. Although multimedia technology has played a pivotal role in many fields, there are still many shortcomings. First of all, the traditional multimedia technology is limited to digital audio, Flash animation, etc. played by classroom projectors, and there is a problem of resource singularity. Secondly, information technology and the Internet are developing at a rapid pace, accompanied by massive data, and the selection of effective data has become a complex issue. Finally, the Internet has entered thousands of households. The traditional status of learning in the classroom alone cannot help students. A smarter multimedia platform is needed to help students learn independently. The advent of the information society and the popularity of the calculated industry, multimedia technology is increasingly used in all aspects of society. The science technology in school is conducive to visualizing unique content, strong 
static relationship, delivering colorful images, making interesting sounds, and increasing students' interest in educational activities.

The main feature of modern distance education methods is that teachers and students can interact in real time or nonreal time across spaces. This is the most significant difference between modern distance education and traditional education methods, and it is also its advantage. Modern distance education methods have the following advantages: teachers' lectures and students' learning can be carried out at different locations at the same time, and there can be sufficient communication between teachers and students; students can independently arrange learning time and location according to their own needs, freely choose learning content, arrange the study plan by oneself, ask the questions in study at any time, and get answers in time; modern distance education methods are conducive to individualized study. In today's era of big data, big data technology can help people get the information they need more easily from massive amounts of data. With the development of smart grids, the distribution system is generating more and more data. In response to this problem, Zhang et al. [19] used big data technology to process and analyze power data, tap potential value, and improve power management. Li [20] introduced big data technology into the logistics field, analyzed logistics information, and predicted market changes, thus helping the logistics industry to further expand smart marketing. Wang and Wang [21] used the data mining and other techniques to construct a systematic framework of TCM physique big data in the field of TCM constitutional science and found the academic laws of TCM constitution used to guide clinical practice. Liu [22] takes the vocational colleges as an example and introduces the technology into the construction of smart campuses to improve skills [23]. Lin and Zhang [24] applied big data technology to radio and television monitoring, improved the intelligence of the satellite radio and television monitoring system, and greatly reduced the workload of manual operations. Ma and Dai [25] helped the coal industry to develop steadily by building a coal big data platform and integrating skill. In summary, big data technology succeeds in various parts to help the industry develop healthily. Therefore, we can consider combining big data technology with multimedia teaching that improve the level of education in China.

Distance education goes through three historical stages: correspondence education, radio and television education, and modern distance education. Modern distance education is a new type of education method that has emerged with the development of modern information technology. The development of computer technology, multimedia technology, and communication technology, especially the rapid development of the Internet, has made a qualitative leap in the means of distance education, and it has become distance education under high-tech conditions. Modern distance education is based on modern distance education methods, including face-to-face, correspondence, and self-study teaching methods, and a variety of media optimization and organic combination of education methods. To solve the problems of multimedia skill from classroom teaching time and space restrictions, teaching resources constraints, and Internet resource confusion, this paper uses $\mathrm{B} / \mathrm{S}$ architecture and streaming media transmission technology to make a personalized network. Through the use of intelligent multimedia technology based on big data, the built system not only has the functions of basic user information management, video resource playback, uploading and downloading, online communication, online testing, etc. but also enables personalized learning resource recommendations based on the learning habit data that the user exhibits while using the system. The system integrates various effects required for production, process, and quality control by combining information technology, physical storage technology, graphical user interface, artificial intelligence technology, data synchronization architecture, and other related fields and delivers image information to customers, through the Internet or other notification methods, promptly and accurately mentions all kinds of users in the form of graphics and text and status, and notifies relevant users as soon as possible.

\section{Method}

2.1. Big Data and Data Mining. There are three characteristics of big data. First, the relative data is mainly large in capacity and can reach the PB level $(1 \mathrm{~PB}=1024 \mathrm{~TB})$. Secondly, the amount of the data is big. Third, data processing is fast. The core strength of big data technology lies in capabilities. The processing modes of big data mainly include directly entire data collection, high efficiency, and main mining of new things, but no systematic method theory has been formed. Data mining is also called knowledge discovery in the database. It refers to the process of revealing hidden and previously unknown data from a large amount of data in the database and discovering valuable information.

Data mining, first of all, needs to collect data according to the task, then transform the data into the required standard format, then use the data mining algorithm to model, and finally display the analyzed results. The specific process is as follows:

(1) First of all, the data collection needs to be carried out. The objects to be studied need to be selected according to the plan, and the information to be collected is positive for the analysis work and may be data of different dimensions or data of different formats.

(2) Save the previously collected data in a unified manner, you can choose to use the database, and you can also use a text file.

(3) Among the collected data, there are often some columns of information that are meaningless to the mining process. One aspect is not related to the problem that needs to be solved. The other aspect is that when the variance of a column of numerical features is zero or very close to zero, this information is of no value. Then, when the data is removed, there is no impact on the results of data mining, and the 
amount of calculation is also reduced. This process is also a convention.

(4) According to the data, the data was not filled when it was saved, resulting in some data missing features, or adding some extraneous features when saving the data. These need to be "cleaned up" to ensure the consistency of the data format. Only in this way can the data be modeled in the later stages to ensure success.

(5) Modeling results have a great relationship with the selection of numerical dimensions. For example, in logistic regression, if the data dimension is large, it can directly affect the results of the model. Then, in order to solve this problem, it is necessary to transform the data into a suitable form, such as normalizing the data $0-1$, letting the data be in the range of $0-1$, and then analyzing the data.

(6) According to the nature of the task, choose the appropriate algorithm to analyze and model the problem. If it is a classification problem, use algorithms such as logistic regression or decision tree. In advanced cases, you can use random forest or promotion methods. If it is a clustering problem, you can try the $K$-means algorithm or the DBSCAN algorithm.

(7) The results of the modeling analysis are reapplied to practice, and it is judged whether the model results are realistic and have a positive effect. Otherwise, the problem needs to be reanalyzed and modeled again.

(8) Once the final mining results are available, the results can be visualized if they can be displayed.

2.2. Improved K-Means Clustering Algorithm. Cluster analysis is to group a series of unmarked data, and these data are all numerical, and according to the distance between the data, the near-distance data is divided into a group, so as to describe the data. The existing $K$-means clustering algorithm adopts a random selection method when selecting the cluster center. This method will produce large errors for the clustering results and affect the correctness of the clustering results. Therefore, this topic improves the $K$-means algorithm to eliminate the impact of randomly selecting cluster centers.

When analyzing the user behavior data, the index is basically consistent with the original $K$-means clustering algorithm. The difference is that the selection idea of the cluster center is different. The algorithmic process of the improved $K$-means clustering algorithm is as follows:

(1) Arbitrarily select a point in the data set as the first cluster center $\mathrm{C}_{1}$.

(2) Calculate the distance from the noncluster center point to each cluster center, and record the distance between the nonclustered center and its nearest cluster center as $D(x)$.

(3) Calculate the probability $D(x)^{2} / \sum_{i=1}^{n} D\left(x_{i}\right)^{2}$ that each nonclustered center becomes the center of the next cluster, and select the next cluster center by the roulette method.

(4) Repeat steps (2) and (3) until $K$ cluster centers are selected.

(5) Calculate the distance from each nonclustered center to $K$ cluster centers, and divide it into the cluster where the nearest cluster center is located.

(6) Recalculate the cluster center for the $K$ clusters, i.e., the centroid of the cluster.

(7) Repeat steps (5) and (6) when the number is enough, or the cluster center position change is less than the set threshold.

It can be seen from the above algorithm process that the first four steps ensure that the distance between the selected $K$ cluster centers is as far as possible, and the error caused by the $K$-means algorithm randomly selecting the cluster center is reduced. It is the step of the existing $K$-means algorithm. Finally, the algorithm is used to analyze the behavior of student users.

2.3. Streaming Media Technology. Virtual reality technology is a significant part in current science space. It is in office design and industry, education and training, military and outdoor services, telemedicine and surgery, sports and games, and space expenditures and has had a significant impact on the production and life of human society. This is a high-tech machine with strong growth and full of vitality. Multimedia network teaching is inseparable from the playback of audio and video. Most users nowadays generally use the method of transmitting while playing audio and video. Streaming media technology is actually playing video data in real time while downloading. By storing the multimedia files containing a large amount of information in the computer memory in advance, the client only needs to wait for a certain buffer time during playback, which not only saves the client's traffic but also saves the customer's viewing time.

In general, the development and application of a technology must be based on certain technical principles, and the principle of streaming media technology is the application of buffer technology. When the client transmits files, the network cannot synchronize the files due to the discontinuity of the data packets. Therefore, the video and audio file formats are generally compressed before being transmitted, and the data is divided into small data packets that can be transmitted. It must be noted here that, due to the dynamic nature of the network, it may cause confusion in the transmission of data packets, resulting in inconsistent timeto-order of file arrivals, so it is necessary to use buffer technology to solve this problem. The buffer system not only regulates the transmission order of the data packets but also enables high-quality continuous decompression and output of the video and audio files compressed before transmission. This method can reduce the playback delay without affecting playback. When the streaming media file is played, the space occupied is not large. The data stored in this buffer is 
generally discarded after being played, so as to free up buffer space for the content that has not been played.

The implementation of streaming services consists of the following:

(1) Streaming data source server.

(2) The video player is used for format conversion of multimedia on the system side, so that the video information is displayed on the web page.

(3) The streaming media server is responsible for storing and transferring the media information that has been formatted.

(4) The protocol layer uses the multimedia transmission protocol commonly used in the network.

(5) The web-side multimedia player provides a variety of operations for providing users with corresponding multimedia files.

In the development process of this system, multimedia transmission uses streaming media transmission technology. One of the basic protocols of streaming media transmission technology is the IP protocol, which implements streaming by sending packets in packets. The transmission process is as follows: first, the multimedia file is compressed, and after the format is converted, it is converted according to the semantics of the IP protocol and then transmitted by grouping, packing, and protocol. After the encoding and decoding of the web page program, the video information is restored and displayed. This can guarantee the integrity of the data and the quality of the multimedia files to a certain extent.

\section{Simulation}

3.1. Overall System Design. Multimedia is an important material foundation of the information society. Multimedia is ubiquitous. Human activities will depend on multimedia to a large extent. The education in the new century cannot be separated from the multimedia. The education model based on multimedia impacts the traditional education model and will become the mainstream education form in the new century. In our traditional teaching process, the most classic teaching mode is based on the teacher, the teacher speaks on the podium, and the students listen in the seat. Another mode of traditional education is the distance education mode such as correspondence education and radio and television education. According to the various functional requirements of the personalized network teaching system, the system mainly includes four functions. And the system combines teacher information management, student information management, and course information management; the teaching resource management module mainly includes teaching video uploading, downloading, and data statistical analysis; the interaction module includes online communication, problem discussion, message recording, etc.; online test management includes test management, online test, test score query, and so on. The overall functional structure of the system is shown in Figure 1.
3.2. System Interface. Because the content of the article is limited, only some interfaces of the multimedia network teaching system are listed here.

3.2.1. Login Interface. All the latest information technology methods optimize the education and teaching process and have achieved remarkable results. The current new information technology revolution marked by multimedia and network has caused profound changes in the field of education and will make a major leap in the education system and teaching mode. At present, online teaching is mainly through online virtual classrooms, campus networks, and the Internet. Web-based multimedia CAI courseware is a combination of multimedia CAI courseware and Web technology, and it will become the main form of multimedia CAI courseware for network teaching.

In order to facilitate the use of the system by teachers and students, the username and password of the system are imported by the school educational administration system. The username and password are the same as the passwords of the teachers and students in the school administration management system. If the username and password are correct, click the "Login" button to log in to the system; otherwise, you will not be able to login. When the user does not want to log in or wants to reenter the account password, click the "Cancel" button to clear the entered information. The login interface of the system is shown in Figure 2.

3.2.2. Video Upload Interface. Online teaching is a "digital learning environment" based on computer, multimedia, network, hypertext, and hypermedia technology. Online resources (teaching content) organize and manage information through the hypertext interconnected "chain" and "section", which promotes the cognition of the meaning of the subject's knowledge. Constructing a nonlinear, net-like, multilevel way of organizing and managing information in online hypertext is more in line with human thinking characteristics and reading habits. The basic structure of hypertext is composed of "Node" and "Link."

In the teaching resource management area, the visible and related lecture videos that the teacher can make are uploaded to the system through the upload function, and the students can select the relevant teaching resource information according to their needs and download them. At the same time, the data statistics function is set in this module, which can count the access status and download status of each teaching resource. Statistics can be used in learning points of students. After logging in to the multimedia network teaching system, the user enters the system home page and selects the video resource management function. Enter the video function management interface, and the interface displays the video resources that can be managed under the user authority. Users can select the video resources they need to manage. Take the video upload interface as an example, as shown in Figure 3.

After logging in to the multimedia network teaching system, the user selects the video resource uploading function, enters the video resource uploading page, inputs 


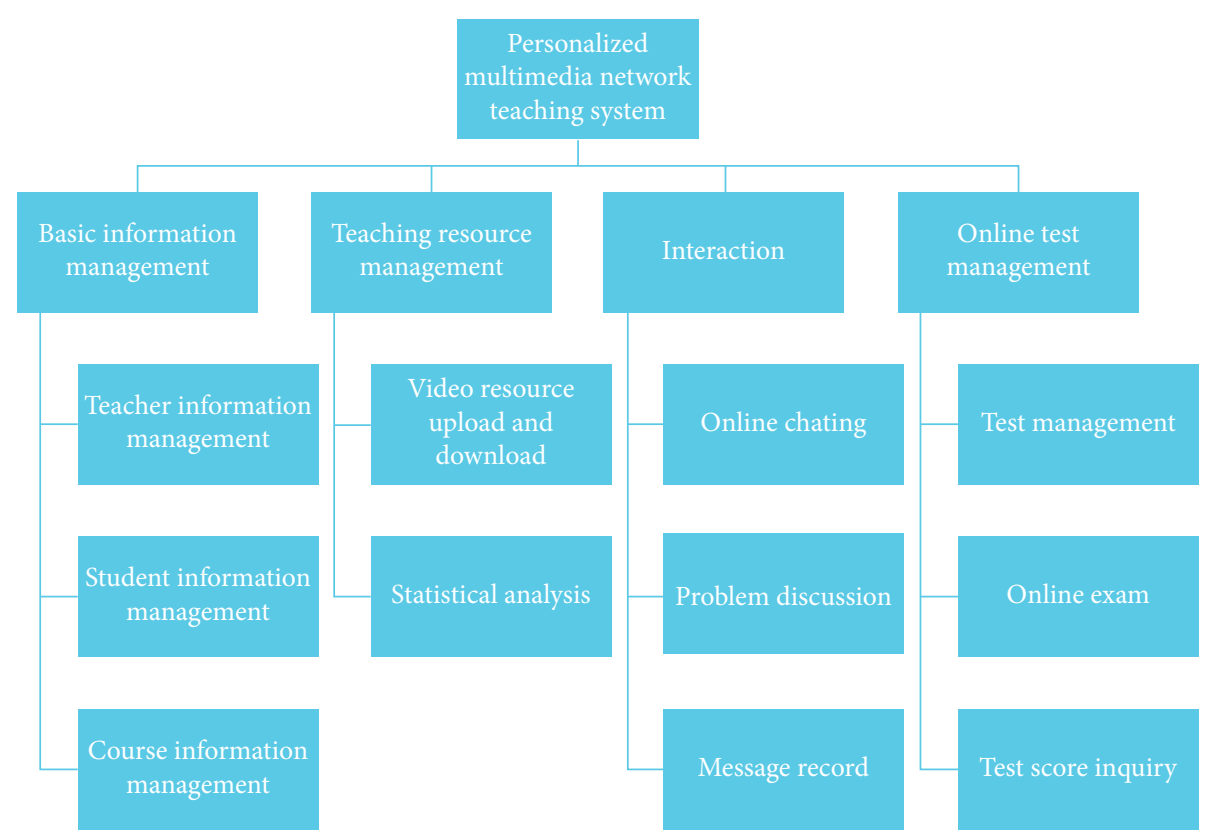

FIGURE 1: System overall functional structure diagram.

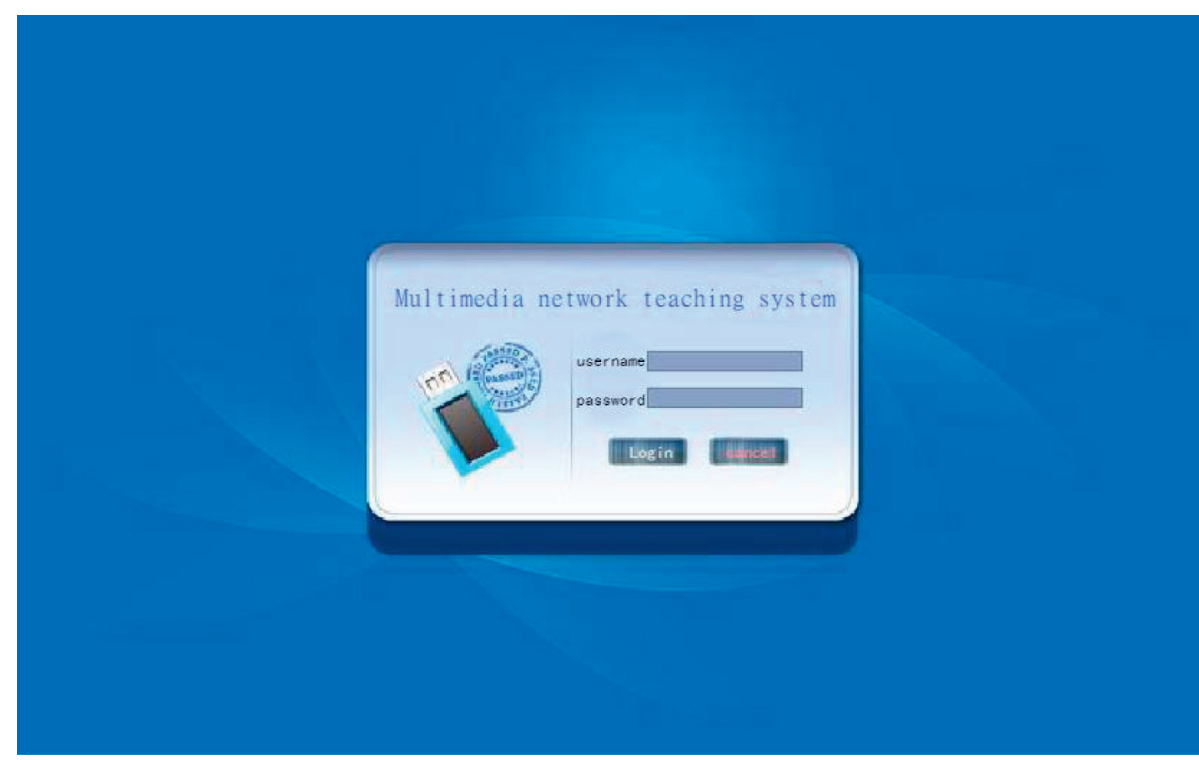

FIGURE 2: Login interface.

the number, name, and the course of the uploaded video resource, clicks the "Browse" button, pops up the local file selection interface, and selects the uploaded video file. Click the "Upload" button to complete the upload of video resources. All teachers and students who log in to the system can browse and view the uploaded video resources.

3.2.3. Online Communication Interface. The requirements for MDBMS can include several aspects: it should include the functions of the standard database management system that should be included in the conventional database, including persistence, concurrency, recovery, and ad hoc query: the modeling facilities involved in multimedia applications, that is, the data model requirements, are database object sharing and operation requirements. The big problems faced by such databases are data retrieval, security, and consistency issues.

After logging in to the multimedia network teaching system, the user enters the system home page. Take online communication as an example, as shown in Figure 4. The user selects the online communication function and enters the online communication page, which has an information input box, an information display module, and an online person module.

Because each student's online communication problems are different, through the preservation of message records, the data mining cluster analysis method is used to recommend personalized learning resources to students. 


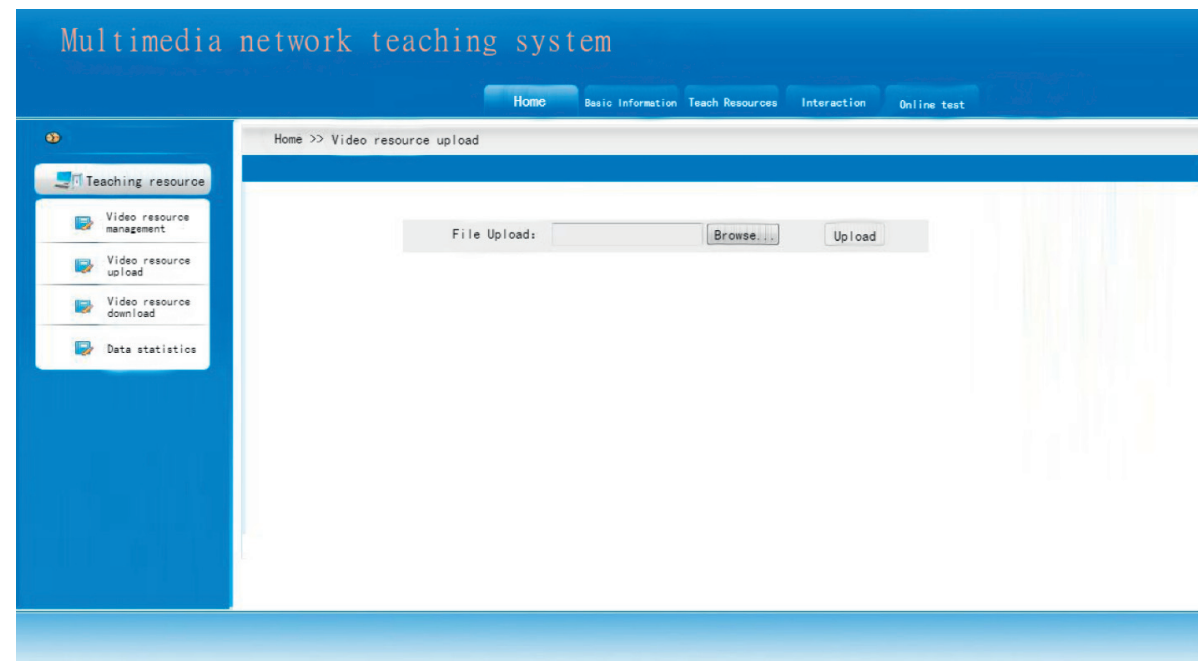

FIGURE 3: Video upload interface.

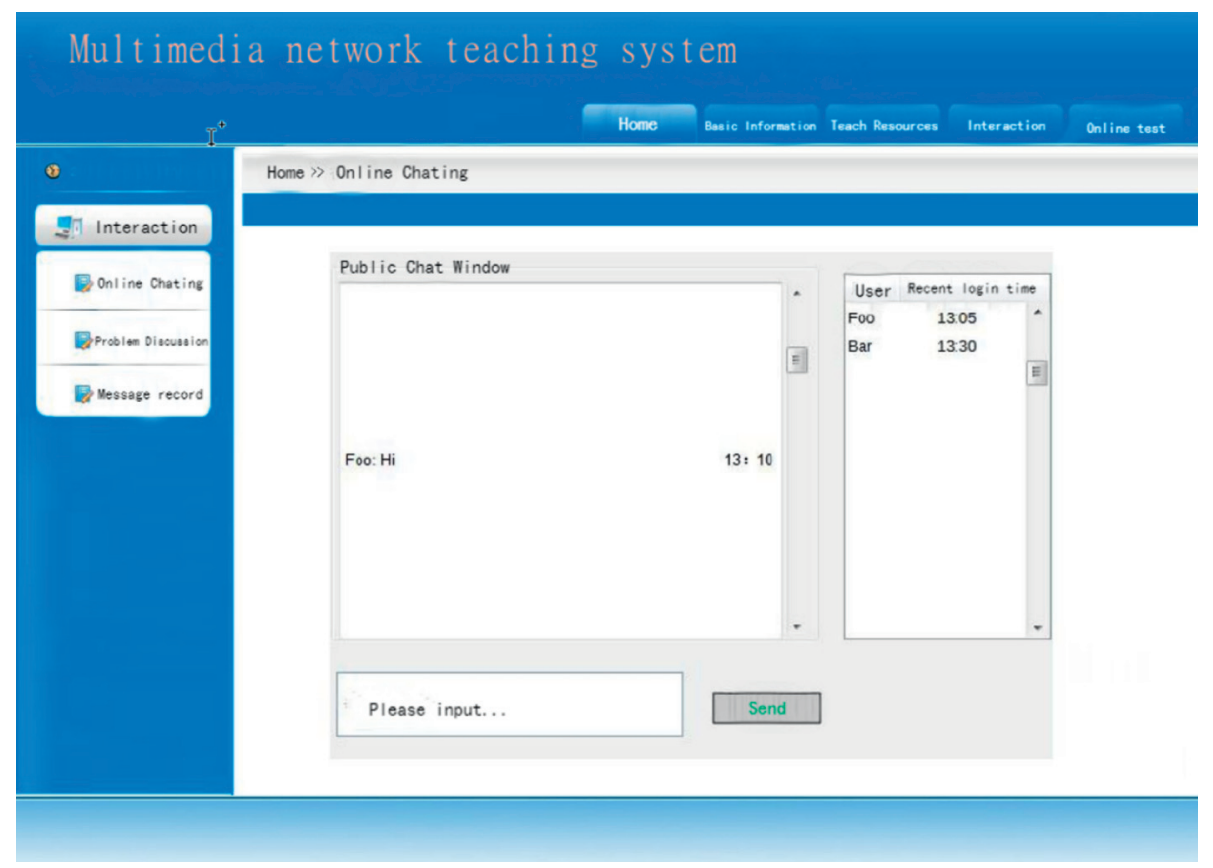

FIgURE 4: Online communication interface.

\section{Results and Discussion}

4.1. Result 1: Video Play. In the information age of the Internet, our educational training goal is to cultivate knowledge-based talents who are creative, good at innovation, and daring to innovate. Cultivating students' innovative ability has become the development trend of today's world. As a teacher, you must not only impart limited knowledge to students but, more importantly, teach students how to learn, so that they have the ability to acquire knowledge, analyze knowledge, apply knowledge, and innovate knowledge, which also meets the requirements of quality education.

The playing interface of the animation design students use in the system to watch related teaching videos is shown in Figure 5. Users can drag the progress bar to select the clips they want to watch. At the same time, you can download the video from the website to the local device while playing the video.

Online education will trigger the "fission" of the three cornerstones of reading, writing, and calculation in the traditional education model. The linear reading method will be replaced by the nonlinear hypertext reading method, and in the form of information, it will be expressed in various media such as text, sound, graphics, and animation; writing will mainly use computers such as keyboard, mouse, and scanning to complete; in terms of computing ability training, more attention will be paid to cultivating students' ability to use computers to solve computing problems, thereby improving the efficiency of problem solving; information technology, information processing, etc. need to be added to the teaching content. In the early development of artificial intelligence, 


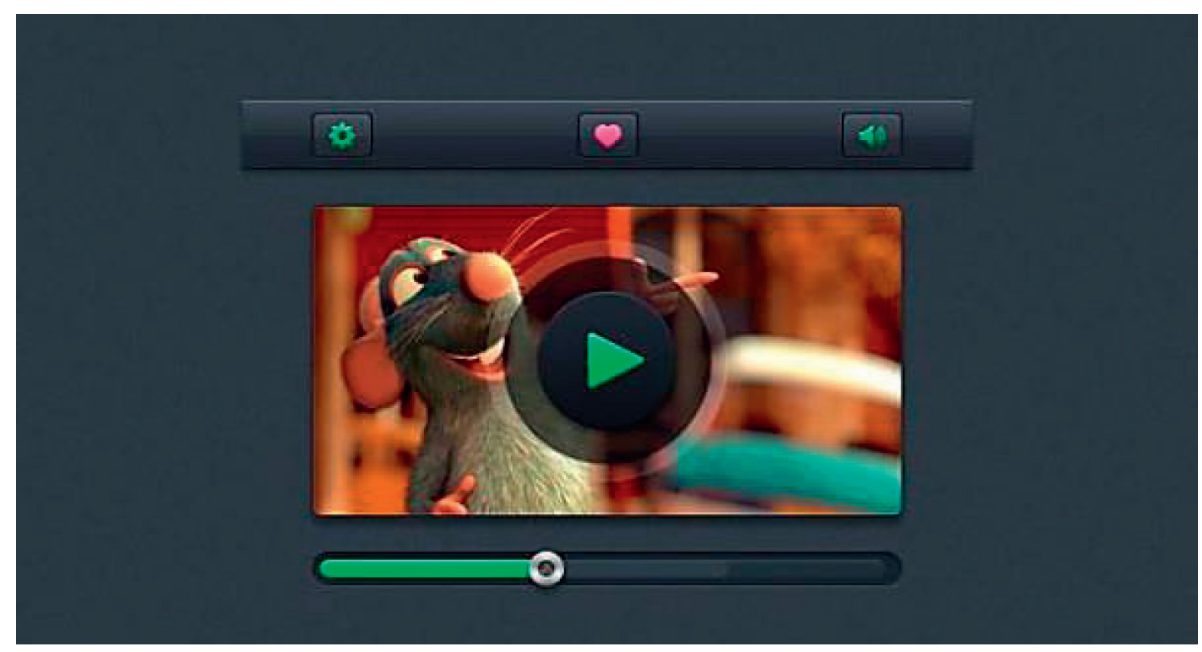

FIGURE 5: Video playback.

semiotics occupies a strong position, because its right is to imitate human thoughts, which is consistent with the first concept proposed by artificial intelligence theory, so we take it as a good example to accept. Due to the use of multiple technologies for verification, the current work of artificial intelligence is reasonable and accurate. Semiotics proposes physical labels and expert programs, which makes the subject of artificial intelligence change from motion to technology for the first time. In use, the video is played smoothly and there is no jamming. The streaming media file transfer process is to first play the data from the server link, then obtain the relevant play parameters through the web page, and finally play the video and audio. The reason why the video can be played while downloading the video is because of streaming technology. Streaming media playback methods mainly include unicast and broadcast. Later, due to the shortcomings of the two, multicast was generated. This topic uses the multicast method. The unicast method will cause data redundancy. The client memory is seriously insufficient. Over time, the server is overwhelmed. If the playback response time is too long, playback will fail or playback will stop. When upgrading hardware or software for these problems, the cost of playback will increase, and the problem of serious server load will not be solved. Although the broadcast solves the problem of the unicast server load capacity, a data packet can be sent to multiple users. But its most serious drawback is that it does not need to be passively received by other customers, and it cannot be effectively controlled, and the playback process is not controlled. This not only wastes a lot of network bandwidth but also causes serious user troubles. In the multicast mode, a single server can be configured with multiple channels to transmit streams for multiple users in need. Users who do not need them are not selected and can use network bandwidth in parallel to improve usage efficiency.

4.2. Result 2: Learning Resource Recommendation. In an open network environment, students and teachers can share resources through the Internet, discuss issues together, and form a collaborative learning method. Through discussion groups, students can express their own opinions or learn from others' perspectives, acquire knowledge, and form a cooperative learning method. In addition, students can obtain information directly from experts other than teachers, which cultivates students' divergent thinking and creative thinking, thereby fostering students' sense of innovation which is conducive to students' innovative ability form.

Through the application of data mining method and improved $K$-means clustering method, the students of animation design majors are analyzed in the video resource clicks, online chat records, etc., and the learning resources are recommended for the corresponding students. The longer a student uses the system, the more data information he or she leaves, and the accuracy of the system in personalized recommendations will change accordingly. The recommended accuracy rate as a function of time is shown in Figure 6. The abscissa indicates the first, third, fifth, seventh, ninth, and eleventh months of the system, and the ordinate indicates the recommended accuracy.

Everything has its two sides. While seeing the positive effect of the Internet on education, we cannot ignore its negative impact on students' thinking and behavior. In online education, the traditional teacher-centered indoctrination learning model is transformed into a student-centered, autonomous learning model. In this model, students rely on their own power to acquire knowledge and give feedback to teachers through homework. Teachers act as instructors to help students achieve their predetermined goals.

In Figure 6, we found that, in the first 5 months, the rate of the recommended resources of the system to the user is relatively fast. In the next few months, although the recommendation accuracy rate continued to rise, the trend slowed down noticeably. According to the survey of the user's return visit and the clustering algorithm improved in this paper, it is found that the reason for this trend is that, at the beginning, the user clicks on various resources and chat information on the platform rarely. Therefore, the computer can mine less useful information, resulting in low recommendation accuracy. In the later stage, due to the accumulation of huge data volume, the user's learning habits and 


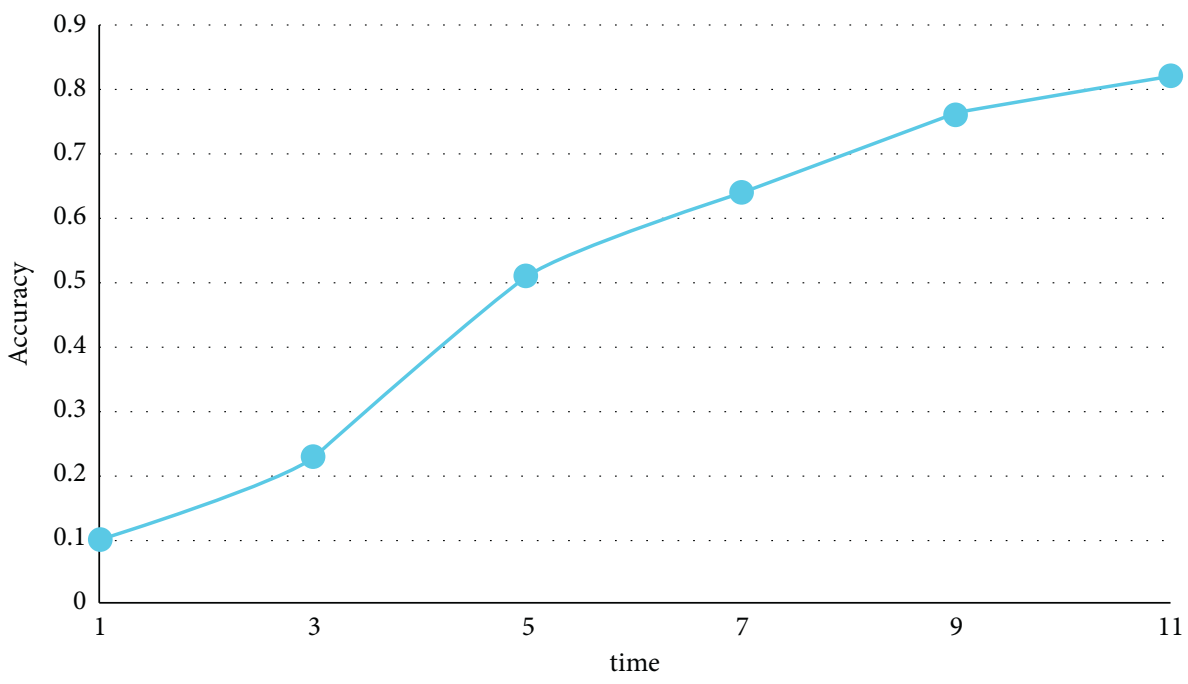

Figure 6: Recommended accuracy rate over time.

styles are all mined, so that the user's preferred resources can be more accurately recommended to the user.

\section{Conclusions}

Although online multimedia have broadened the learning content of students, students have to sit in front of the screen and learn, and they lose the opportunity to contact with society, life, and practice. Some students are obsessed with computer games, which affects the problem of learning. In the Internet multimedia, various cultures are mixed, various information is mixed, and bad information will corrode the students' ideology and morality. These kinds of problems have issued new challenges to our education. With the advent of the era of big data, how to quickly and effectively extract the information of interest from users is a hot topic. By mining data and combining intelligent multimedia technology, useful information can be visualized. The scale of education in China is constantly expanding, and modern education and teaching methods will help improve the education level in China. The multimedia network teaching system constructed in this paper relies on the Internet to complete the transmission of educational teaching resources and related information. It is a basic network system that combines multimedia and streaming media technologies to add rich multimedia capabilities to the system. The system disseminates various types of media information, processes the media information, and shares the information, thus forming an idealized multimedia network teaching environment. Although the research in this paper has achieved certain results, due to time constraints and other issues, there are still the following deficiencies:

(1) The function is not perfect and is too general. Although the interface conforms to the principle of simplicity, it is not friendly enough.

(2) In terms of personalized recommendation, the accuracy of recommendation needs to be further improved. It is possible to further recommend various learning resources, analyze the learning situation of students, and help students further improve their learning level.

(3) Data transmission accuracy and system stability need to be further improved.

\section{Data Availability}

This article does not cover data research. No data were used to support this study.

\section{Conflicts of Interest}

The author declares no conflicts of interest.

\section{References}

[1] L. Wang, "Discussion on the application of information technology in classroom teaching in secondary vocational schools," Journal of Multimedia and Network Education (Electronic Edition), no. 6, pp. 245-246, 2017.

[2] Y. Yang, "Analysis on the effective application of multimedia in secondary school mathematics classroom in the new period," Education Modernization: Electronic Edition, no. 22, p. 139, 2017.

[3] W. Song, "Analysis of education management innovation under the background of big data," Education Modernization, vol. 5, no. 20, pp. 304-305, 2018.

[4] Y. Fan, "Discussion on the teaching mode of the high school Chinese papers based on big data," Examination Weekly, no. 51, p. 50, 2018.

[5] W. Ma, "Research on information teaching model based on big data era," Winning the Future, no. 1, p. 2, 2017.

[6] X. Luo, "Using computer multimedia technology to improve teaching efficiency," Rural Economy and Technology, vol. 28, no. s1, p. 226, 2017.

[7] Y. Wu, "Development and integration of virtual reality multimedia teaching resources based on cloud platform," Examination Weekly, no. 61, p. 132, 2017. 
[8] L. Wang, "Application and enlightenment of multimedia technology in teaching," Administrative Assets and Finance, no. 8, p. 90, 2017.

[9] X. Zou and Y. Zhou, "Application of authorware-based multimedia courseware in sandbox teaching of enterprise production and management," Modern Vocational Education, no. 12, p. 121, 2017.

[10] Y. Wang, "Application of multimedia teaching led by case name analysis in the teaching of new nurses in the operating room," Journal of Traditional Chinese Medicine Management, vol. 26, no. 9, pp. 30-31, 2018.

[11] J. Chen and X. Liu, "Application of computer multimedia technology in auxiliary physical education," Journal of Automation \& Instrumentation, no. 2, pp. 129-130, 2017.

[12] L. Wang, "Analysis of the integration of multimedia technology and junior middle school English teaching," Academic Journal, no. 3, pp. 239-240, 2017.

[13] L. Binbin, "Multimedia technology optimization of junior middle school english reading teaching," English Pictorial (Advanced Edition), no. 2, pp. 97-98, 2017.

[14] L. Chen, "Application practice of multimedia technology in high school physics teaching process," Chinese Journal of School Education, no. 1, pp. 164-165, 2017.

[15] Y. Wang, "Application of multimedia technology in high school chemistry teaching," New Curriculum, no. 24, p. 114, 2017.

[16] Z. Lv, R. Lou, J. Li, A. K. Singh, and J. Li, "Big data analytics for $6 \mathrm{G}$-enabled massive internet of things," IEEE Internet of Things Journal, vol. 8, no. 99, p. 1, 2021.

[17] Z. Lv, Y. Han, A. K. Singh, and G. Manogaran, "Trustworthiness in industrial IoT systems based on artificial intelligence," IEEE Transactions on Industrial Informatics, vol. 17, no. 99, p. 1, 2020.

[18] Q. Yue, "Application of multimedia technology in primary school music teaching," New Campus, no. 2, p. 146, 2018.

[19] D. Zhang, J. Wang, and X. Liu, "Application of big data technology in power system," Power Supply, vol. 32, no. 8, pp. 6-11, 2015.

[20] Z. Li, "Big data technology leads smart marketing in logistics industry," Economic Research Guide, vol. 366, no. 16, pp. 66-67, 2018.

[21] J. Wang and Q. Wang, "Thoughts on the application of big data technology in TCM constitution," Chinese Journal of Traditional Chinese Medicine, no. 3, pp. 22-24, 2017.

[22] S. Liu, "Application of big data technology in the construction of smart campus in higher vocational colleges," Network Security Technology \& Application, no. 3, p. 127+129, 2017.

[23] E. Mohamed, "The relation of artificial intelligence with internet of things: a survey," Journal of Cybersecurity and Information Management, vol. 1, no. 1, 2020.

[24] L. Lin and C. Zhang, "Application of big data technology in broadcast and television monitoring," Radio and TV Information, no. 6, pp. 96-99, 2017.

[25] X. Ma and W. Dai, "Research status and application prospect of big data technology in coal industry," Industry and Mine Automation, vol. 44, no. 1, pp. 54-58, 2018. 